\title{
When the Poisoned Risk Poisoning Others: Fatal Sodium Azide Overdose
}

Marcin M. Ciesla; Diane P. Calello, MD; Lewis S. Nelson, MD

\section{A 24-year-old man arrived at the ED in cardiac arrest after intentionally ingesting sodium azide.}

\section{Case}

A 24-year-old man in cardiac arrest was brought to the ED via emergency medical services (EMS). Unfortunately, resuscitation efforts were unsuccessful. Little was known about the patient, but the emergency physician was informed that the patient had ingested sodium azide $\left(\mathrm{NaN}_{3}\right)$, which he had ordered online. The patient collapsed shortly after ingesting the sodium azide, approximately the same time police officers arrived at the patient's home.

No specific details were known about the patient's ingestion. Upon learning of the exposure to sodium azide, a member of the ED staff contacted the local poison control center for information on the proper course of action to ensure staff safety and limit exposure. Shortly thereafter, several of emergency medical technicians and police officers, who had responded to the emergency assistance call for this patient, presented to the ED with concerns of exposure.

\section{What is sodium azide?}

Sodium azide is a colorless, odorless crystalline water-soluble solid that has a pK of 4.8. ${ }^{1}$ When sodium azide is dissolved in an acid, it liberates hydrazoic acid $\left(\mathrm{HN}_{3}\right)$, which has a pungent odor, high vapor pressure $(484 \mathrm{~mm} \mathrm{Hg}$ ), and a relatively low-boiling point of $37^{\circ} \mathrm{C}\left(98^{\circ} \mathrm{F}\right) .^{2}$

The most common industrial use of sodium azide is as a propellant in air bags. In this capacity, sodium azide rapidly decomposes to nitrogen gas when it reaches a temperature of $300^{\circ} \mathrm{C}\left(572^{\circ} \mathrm{F}\right)$, causing rapid expansion of the air bag. In addition to air bags, sodium azide is used in research laboratories as a preservative and in agriculture as a pesticide. The main nontoxicological concern with all azide agents is the potential for explosion when they react with metals, such as lead, copper, silver, and mercury, to form metal azides that are sensitive to shock. ${ }^{3}$ An example of the explosive nature of these azides was demon-

\footnotetext{
Mr Ciesla is a medical student, Rutgers New Jersey Medical School, Newark. Dr Calello is an associate professor of emergency medicine; and executive and medical director, New Jersey Poison Information and Education System, Rutgers New Jersey Medical School, Newark. Dr Nelson, editor of "Case Studies in Toxicology," is professor and chair of the department of emergency medicine, Rutgers New Jersey Medical School; and a medical toxicologist, New Jersey Poison Information \& Education System, both in Newark. He is also associate editor, toxicology, of the EMERGENCY MEDICINE editorial board.
}

Authors' Disclosure Statement: The authors report no actual or potential conflict of interest in relation to this article. 
strated in a report wherein diluted sodium azide was poured down a drain, causing an explosion as a worker was fixing the pipe. ${ }^{4}$

In addition to industrial and commercial use, sodium azide is occasionally used in suicide attempts because it is rapidly fatal, has no specific antidote, and can be purchased online. $^{3}$

\section{What is the toxicity of sodium azide?}

The lethal dose for both oral and dermal exposure to sodium azide is approximately 10 to $20 \mathrm{mg} / \mathrm{kg}^{3{ }^{3,5}}$ Therefore, ingestion of 700 mg of sodium azide, a volume approximately the size of a penny, is likely to be fatal. ${ }^{3}$

Sodium azide is primarily a mitochondrial toxin, which binds the electron transport chain, inhibiting oxidative phosphorylation. The resulting reduction in adenosine triphosphate (ATP) production, even in the presence of oxygen, results in metabolic failure. ${ }^{6}$ This mechanism of action is similar to that of cyanide, although sodium azide causes more pronounced vasodilation due to the in vivo conversion of some azide to the vasodilator nitric oxide. ${ }^{7}$ Some reports suggest that azide lethality is due to enhanced excitatory transmission from nitric oxide in the central nervous system. ${ }^{8}$

\section{What are the clinical manifestations of azide poisoning, and what is the treatment?}

The early clinical findings of a patient with azide poisoning include hypotension, dizziness, headache, nausea, vomiting, palpitations, tachycardia, dyspnea, and restlessness. Inhalation of hydrazoic acid can also produce wheezing and coughing. The most common effect is hypotension, which can occur within 1 minute of exposure. Following depletion of cellular ATP, anaerobic glycolysis generates lactate and produces acidemia. More severe findings of azide poisoning include seizures, cardiac arrhythmia, loss of consciousness, pulmonary edema, and cardiopulmonary failure. ${ }^{3}$

Currently, there is no specific antidote for azide poisoning, and treatment mainly consists of supportive care. Cyanide anti- dote treatments are generally ineffective in reducing azide-related death in animal models. ${ }^{3,8}$ Early aggressive supportive care can improve survival rates. ${ }^{9}$ Some authors suggest that administration of oral activated charcoal, orogastric lavage, hemodialysis, and plasma exchange reduce azide concentrations, while others believe these treatments have little effect. ${ }^{3,9}$ More research is needed to identify effective therapeutic measures and to control for dose, time, and patient population.

What are the safety concerns for emergency medical technicians and hospital staff following exposure to sodium azide?

The most probable routes of exposure for prehospital and hospital staff include dermal contact with sodium azide or inhalation of gaseous hydrazoic acid; inhalational exposure is most concerning. ${ }^{1}$ In one case, hospital-staff members developed headaches, light-headedness, and nausea while treating a patient for azide poisoning; however, staff exposure was not confirmed and no sequelae were evident. ${ }^{10}$

More objectively, workers at an azide plant exposed to azide concentrations above the occupational exposure limit developed headaches, hypotension, and palpitations. ${ }^{11}$ Another study found no evidence of kidney, heart, or liver damage after patients were given sodium azide for more than a year during a clinical trial. ${ }^{12}$ Not unexpectedly, there is little risk of exposure when proper safety precautions are taken.

Emergency response personnel should carefully inspect the scene for the presence of any sodium azide powder, and should also question bystanders and family members to determine if anyone performed mouth-tomouth resuscitation on the patient. Standard universal precautions, along with attentiveness to one's surroundings, should be sufficient to prevent dermal exposure. If small amounts of sodium azide residue are found on the patient, his or her clothes should be cautiously removed and placed in a plastic bag to prevent dispersion of particles. If large 
quantities of sodium azide are present on a patient, the hazardous materials response team should be called, in accordance with institutional and regional protocols. To avoid explosion, every attempt should be made to prevent azide salt (eg, from emesis) from contact with any metal surfaces (eg, oxygen tanks, metal stretcher). ${ }^{13}$

Vomit from patients who have ingested sodium azide can cause liberation of hydrazoic acid, which can escape through the esophagus. A pungent ambient odor may provide a warning, which is particularly concerning in a confined space such as an ambulance. As a precaution, EMS personnel should open windows and maximize ventilation. After the call, EMS and hospital personnel should thoroughly wash their hands with soap and water, and change their uniform if they believe it has been contaminated. There is no risk of delayed exposure following exposure to hydrazoic acid.

During autopsy, medical examiners must exercise caution due to the potential for liberation of hydrazoic acids from the stomach. ${ }^{14}$ Unless it is absolutely necessary, the medical examiner should avoid opening the stomach. If this is unavoidable, the autopsy should occur in a well-ventilated setting with the examiner wearing a supplied air respirator to limit exposure in a high-risk scenario.

\section{Case Conclusion}

None of the exposed first responders experienced dizziness, light-headedness, or irritation, and after a period of observation in the ED, they were discharged home without further sequelae. All hospital staff involved in the patient's care, including those who performed cardiopulmonary resuscitation on the patient and cleaned his room, were advised to use protective equipment when handling the patient and bodily secretions. None of the health care workers developed abnormal clinical findings. Given the hazard in conducting a full postmortem examination, the medical examiner opted to send blood, bile, urine, and vitreous humor out for analysis, but did not conduct a full postmortem examination. Notably, the stomach was not opened, and its contents were not exposed.

\section{References}

1. Compound summary for CID 33557 (sodium azide). National Center for Biotechnology Information. PubChem Compound Database. https://pubchem.ncbi. nlm.nih.gov/compound/sodium_azide. Accessed May 10, 2018

2. Compound summary for CID 24530 (hydrogen azide). National Center for Biotechnology Information. PubChem Compound Database. https://pubchem.ncbi.nlm.nih.gov/compound/hydrazoic_acid. Accessed May 10, 2018.

3. Chang S, Lamm SH. Human health effects of sodium azide exposure: a literature review and analysis. Int J Toxicol. 2003;22(3):175-186. doi:10.1080/10915810305109.

4. Sodium azide explosion hazard. Washington State Department of Labor \& Industries. Division of Occupational Safety and Health. https://www.lni.wa.gov/ safety/hazardalerts/SodiumAzide.pdf. August 11, 2011. Accessed May 10, 2018.

5. Safety data sheet: sodium azide. ThermoFischer Scientific. https://www.fishersci.com/store/msds?par $\mathrm{tNumber}=\mathrm{S} 227 \mathrm{I} 1 \&$ productDescription $=\mathrm{SODIUM}+\mathrm{AZ}$ IDE+GRAN+PURIF+1+KG\&vendorId=VN00033897\& countryCode $=$ US\&language $=$ en. Updated January 17, 2018. Accessed May 10, 2018.

6. Bogucka K, Wojtczak L. Effect of sodium azide on oxidation and phosphorylation processes in rat-liver mitochondria. Biochim Biophys Acta. 1966;122(3):381-392. doi:10.1016/09266593(66)90031-2

7. Kruszyna H, Kruszyna R, Smith RP, Wilcox DE. Red blood cells generate nitric oxide from directly acting, nitrogenous vasodilators. Toxicol Appl Pharmacol. 1987;91(3):429-438. doi:10.1016/0041008x(87)90064-0.

8. Smith RP, Louis CA, Kruszyna R, Kruszyna H. Acute neurotoxicity of sodium azide and nitric oxide. Fundam Appl Toxicol. 1991;17(1):120-127. doi:10.1093/ toxsci/17.1.120.

9. Watanabe K, Hirasawa H, Oda S, et al. A case of survival following high-dose sodium azide poisoning. Clin Toxicol (Phila). 2007;45(7):810-811.

10. Abrams J, el-Mallakh RS, Meyer R. Suicidal sodium azide ingestion. Ann Emerg Med. 1987;16(12):13781380. doi:10.1016/s0196-0644(87)80423-7

11. Trout D, Esswein EJ, Hales T, Brown K, Solomon G, Miller M. Exposures and health effects: an evaluation of workers at a sodium azide production plant. Am J Ind Med. 1996;30(3):343-350.

12. Black, MM, Zweifach BW, Speer FD. Comparison of hypotensive action of sodium azide in normotensive and hypertensive patients. Exper Biol Med. 1954;85(1):11-16. doi:10.3181/00379727-85-20770.

13. Emergency preparedness and response. Facts about sodium azide. Centers for Disease Control and Prevention. Office of Public Health Preparedness and Response. https://emergency.cdc.gov/agent/sodiumazide/basics/facts.asp. Updated April 10, 2018 Accessed May 10, 2018.

14. Le Blanc-Louvry I, Laburthe-Tolra P, Massol V, et al. Suicidal sodium azide intoxication: An analytical challenge based on a rare case. Forensic Sci Int. 2012;221(1-3):e17-20. doi:10.1016/j. forsciint.2012.04.006. 